\title{
BOUNDED AND FULLY BOUNDED MODULES
}

\author{
A. HAGHANY, M. MAZROOEI and M. R. VEDADI ${ }^{凶}$
}

(Received 16 January 2011)

\begin{abstract}
Generalizing the concept of right bounded rings, a module $M_{R}$ is called bounded if $\operatorname{ann}_{R}(M / N) \leq_{e} R_{R}$ for all $N \leq_{e} M_{R}$. The module $M_{R}$ is called fully bounded if $(M / P)$ is bounded as a module over $R / \operatorname{ann}_{R}(M / P)$ for any $\mathcal{L}_{2}$-prime submodule $P \triangleleft M_{R}$. Boundedness and right boundedness are Morita invariant properties. Rings with all modules (fully) bounded are characterized, and it is proved that a ring $R$ is right Artinian if and only if $R_{R}$ has Krull dimension, all $R$-modules are fully bounded and ideals of $R$ are finitely generated as right ideals. For certain fully bounded $\mathcal{L}_{2}$-Noetherian modules $M_{R}$, it is shown that the Krull dimension of $M_{R}$ is at most equal to the classical Krull dimension of $R$ when both dimensions exist.
\end{abstract}

2010 Mathematics subject classification: primary 16P60, 16P70; secondary 16P40.

Keywords and phrases: bounded module, FBN ring, Krull dimension, $\mathcal{L}_{2}$-Noetherian module, $\mathcal{L}_{2}$-prime module.

\section{Introduction}

Throughout rings will have unit elements and modules will be right unitary. We use the notations $N \unlhd M, N \leq_{e} M$ to denote respectively that $N$ is a fully invariant, essential submodule of $M$. Hence $N \unlhd_{e} M$ means $N$ is a fully invariant essential submodule of $M$. Recall from [3] that $R$ is a right bounded ring if for every $I \leq_{e} R_{R}$ there exists $B \unlhd_{e} R_{R}$ with $B \subseteq I$. The ring $R$ is called right fully bounded if every prime factor of $R$ is a right bounded ring. Right fully bounded right Noetherian rings (right FBN) and modules over them have been studied extensively and are known to have a number of interesting properties; see, for example, [5-8]. The concept of right bounded rings has been generalized to bounded modules by earlier authors. In [10], modules $M_{R}$ with the property that $R / P$ is a right fully bounded ring for every $P \in \operatorname{Ass}\left(M_{R}\right)$ were called 'bounded' and they were studied when $R$ is right Noetherian [10, Theorem 2.6]. In [6], modules $M_{R}$ with $\operatorname{ann}_{R}(M / N) / \operatorname{ann}_{R}(M) \leq_{e} R / \operatorname{ann}_{R}(M)$, for any $N \leq_{e} M_{R}$, were studied and called bounded. To avoid confusion, we shall say that the latter modules are $L$ bounded, while we define a bounded module $M_{R}$ by the condition $\operatorname{ann}_{R}(M / N) \leq_{e} R_{R}$

(c) 2011 Australian Mathematical Publishing Association Inc. 0004-9727/2011 \$16.00 
for any $N \leq_{e} M_{R}$. Our bounded modules form a class bigger than the class of Lbounded modules. Clearly, a module $M_{R}$ is L-bounded if and only if $M$ is bounded as a module over $R / \operatorname{ann}_{R}(M)$.

We study bounded modules and show that they are invariant under Morita equivalences (Theorem 3.3). It follows that L-boundedness and right boundedness are Morita invariant properties. It is shown that all $R$-modules are bounded if and only if $\operatorname{Soc}\left(R_{R}\right) \leq_{e} R_{R}$ (Theorem 3.5) and, as a corollary, we show that right semiArtinian rings are precisely rings with all modules L-bounded. A module $M_{R}$ is called fully bounded if $(M / P)_{R}$ is L-bounded for any fully invariant $\mathcal{L}_{2}$-prime submodule $P \triangleleft M_{R}$ in the sense of [11]. In [12] $\mathcal{L}_{2}$-prime submodules were called 'fully prime submodules'. The class $\operatorname{Spec}_{2}\left(M_{R}\right)$ of fully invariant $\mathcal{L}_{2}$-prime submodules of a given module $M_{R}$ properly lies between the class of 'prime' submodules of $M_{R}$ in the sense of [2] and the class of prime submodules $N$ of $M_{R}$ in the sense of $\operatorname{ann}_{R}(N)=\operatorname{ann}_{R}(M)$ for any $0 \neq N \leq M$. A characterization of rings with all modules fully bounded is given in Theorem 3.7 and it is shown that a ring $R$ is right Artinian if and only if $R_{R}$ has Krull dimension, all $R$-modules are fully bounded and ideals of $R$ are finitely generated as right ideals (Proposition 3.8). In the last section, we deal with bounded modules with Krull dimension and, for a quasi projective fully bounded $\mathcal{L}_{2}$-Noetherian module $M_{R}$, it is shown that $\mathrm{K} \cdot \operatorname{dim}(M) \leq \mathrm{Cl} \cdot \mathrm{K} \cdot \operatorname{dim}(R)$ when the dimensions exist. A well-known result on right FBN rings $R$ stating that $\mathrm{K} \cdot \operatorname{dim}\left(R_{R}\right)=\mathrm{Cl} \cdot \mathrm{K} \cdot \operatorname{dim}(R)$ may then be obtained by Theorem 4.1; see also [3, Theorem 15.13]. Any unexplained terminology and all the basic results on rings and modules that are used in the sequel can be found in $[1,3]$.

\section{Preliminaries}

We begin by recalling some definitions from [11]. An $R$-module $M$ is called $\mathcal{L}_{2}$-Noetherian if $M$ finitely generates all of its fully invariant submodules and has ascending chain condition (acc) on them. Some examples of $\mathcal{L}_{2}$-Noetherian modules are Noetherian self-generator modules and modules without nonzero fully invariant submodules. Note that the module $R_{R}$ is $\mathcal{L}_{2}$-Noetherian if and only if every ideal of $R$ is finitely generated as a right ideal. A proper submodule $P$ of a module $M_{R}$ is called $\mathcal{L}_{2}$-prime if, for every $W_{1}, W_{2} \unlhd M_{R}$, the condition $W_{1} \star W_{2} \subseteq P$ implies that $W_{1} \subseteq P$ or $W_{2} \subseteq P$ where $W_{1} \star W_{2}=\operatorname{Hom}_{R}\left(M, W_{1}\right) W_{2}$. If (0) is an $\mathcal{L}_{2}$-prime submodule of $M_{R}$ then $M$ is called an $\mathcal{L}_{2}$-prime $R$-module. In the following we present some facts on $\mathcal{L}_{2}$-Noetherian and $\mathcal{L}_{2}$-prime modules for later use.

Lemma 2.1. Let $M_{R}$ be a nonzero module with $M I=0$ for some $I \triangleleft R$.

(i) If $N \unlhd M_{R}$ and $Q / N \unlhd M / N$, then $Q \unlhd M$.

(ii) If $M_{R}$ is quasi projective and $K \leq L \unlhd M$, then $L / K \unlhd M / K$.

(iii) $M_{R}$ is $\mathcal{L}_{2}$-Noetherian if and only if $M_{R / I}$ is $\mathcal{L}_{2}$-Noetherian.

(iv) If $M_{R}$ is $\mathcal{L}_{2}$-Noetherian then $M / N$ is an $\mathcal{L}_{2}$-Noetherian $R$-module for any $N \unlhd M_{R}$. 
(v) If $M_{R}$ is quasi projective, $N \triangleleft M$ and $N \leq P$ then $P / N \in \operatorname{Spec}_{2}(M / N)$ if and only if $P \in \operatorname{Spec}_{2}(M)$.

(vi) If $M_{R / I}$ is bounded then $M_{R}$ is bounded. Consequently, L-bounded and fully bounded $\mathcal{L}_{2}$-prime modules are bounded.

(vii) $M_{R / I}$ is fully bounded if and only if $M_{R}$ is fully bounded.

Proof. (i), (ii) and (iii) follow by routine arguments and (v) follows by part (ii).

To prove (iv), let $N \unlhd M_{R}$ and notice that $M / N$ has acc on its fully invariant submodules by part (i). On the other hand, if $L / N \unlhd M / N$ then $L \unlhd M$ and so $M$ finitely generates $L$ by our assumption. Hence there exists an $R$-epimorphism $f: M^{(n)} \rightarrow L$ for some positive integer $n$. Let $\iota_{i}: M \rightarrow M^{(n)}$ be the natural inclusion map for $i=1, \ldots, n$. Since $N \unlhd M, f \iota_{i}(N) \subseteq N$. This shows that the map $g:(M / N)^{(n)} \rightarrow L / N$ with $g\left(x_{1}+N, \ldots, x_{n}+N\right)=f\left(x_{1}, \ldots, x_{n}\right)+N$ is well defined. Clearly $g$ is also an $R$-epimorphism. Thus $M / N$ finitely generates $L / N$, proving that $M / N$ is an $\mathcal{L}_{2}$-Noetherian $R$-module.

(vi) and (vii) follow by definitions and the fact that if $J / I$ is an essential right ideal of $R / I$ then $J \leq_{e} R$.

In [4], an $R$-module $M$ with $S=\operatorname{End}_{R}(M)$ was called endoprime if $\operatorname{lann}_{S}(N)$ is zero for any $0 \neq N \unlhd M_{R}$. It is easy to verify that retractable endoprime modules are $\mathcal{L}_{2}$-prime. (A module $M_{R}$ is retractable if $\operatorname{Hom}_{R}(M, N) \neq 0$ for any $0 \neq N \leq M$.) We will use the following result to characterize rings with all modules fully bounded.

PRoposition 2.2. The following statements are equivalent for a ring $R$.

(i) $R$ is a prime ring.

(ii) $\operatorname{End}_{R}(F)$ is a prime ring for some free $R$-module $F$.

(iii) $\operatorname{End}_{R}(F)$ is a prime ring for every free $R$-module $F$.

(iv) Every free $R$-module is $\mathcal{L}_{2}$-prime.

Proof. For the equivalences (i) $\Leftrightarrow$ (ii) $\Leftrightarrow$ (iii), note that if $S=\mathbb{C} \mathbb{F} \mathbb{M}_{\Gamma}(R)$, a column finite matrix ring over $R$ for some nonempty set $\Gamma$, and $\left(a_{i j}\right) S\left(b_{i j}\right)=0$ for some matrices $\left(a_{i j}\right),\left(b_{i j}\right) \in S$ then $E_{k k}\left(a_{i j}\right)\left(E_{l l} R E_{r r}\right)\left(b_{i j}\right) E_{t t}=0$ for any $k, l, r, t \in \Gamma$ where $E_{x y}$ is the matrix with 1 as $(x, y)$ th entry and zero elsewhere. It follows that $a_{k l} R b_{r t}=0$ for any $k, l, r, t \in \Gamma$. Thus if $\left(b_{i j}\right) \neq 0$ then we can deduce that $\left(a_{i j}\right)=0$ provided that $R$ is prime ring.

(iii) $\Rightarrow$ (iv). Apply [4, Proposition 1.3(3)] for a free $R$-module $F$. Thus $F_{R}$ is endoprime and hence an $\mathcal{L}_{2}$-prime module.

(iv) $\Rightarrow$ (i). This is clear.

\section{The class of (fully) bounded modules}

We study the class of bounded modules and show that bounded, L-bounded and right bounded modules are Morita invariant properties. Several characterizations of rings $R$ with essential socles are given. In particular, semi-Artinian (respectively Artinian) rings are characterized in terms of L-bounded (respectively fully bounded) $R$-modules. 
Proposition 3.1.

(i) The class of bounded modules is closed under taking submodules, factor modules and finite direct sums.

(ii) If $M_{R}$ is a quasi projective fully bounded module, then $M / N$ is fully bounded for any $N \unlhd M$.

Proof. (i) Let $M_{R}$ be bounded and $N \leq_{e} K \leq M_{R}$. There exists $L \leq M$ such that $K \oplus L \leq_{e} M_{R}$. Thus $N \oplus L \leq_{e} M_{R}$. Since $M_{R}$ is bounded, there exists $I \leq_{e} R_{R}$ such that $M I \subseteq N \oplus L$. It follows that $K I \subseteq N$, proving that $K_{R}$ is bounded.

To prove that $M / K$ is a bounded $R$-module, let $N / K \leq_{e} M / K$. Then $N \leq_{e} M_{R}$ and so there exists $J \leq_{e} R_{R}$ such that $M J \subseteq N$ by our assumption. This shows that $(M / K) J \subseteq N / K$.

Finally, assume that $V=\bigoplus_{i=1}^{k} M_{i}$ where each $M_{i}$ is a bounded $R$-module. Suppose that $N \leq_{e} V_{R}$. It is easy to verify that $N \cap M_{i} \leq_{e} M_{i}$ for each $i$. Thus by our assumption on $M_{i}$, there exists $I_{i} \leq_{e} R$ such that $M_{i} I_{i} \subseteq N$. Let $I=\bigcap_{i=1}^{k} I_{i}$. Then $I \leq_{e} R$ and $V I \subseteq N$, as desired.

(ii) This follows from Lemma 2.1(v).

Corollary 3.2. A ring $R$ is right (fully) bounded if and only if any finitely generated $R$-module is (fully) bounded.

Proof. We only prove the fully bounded case. Assume that $R$ is a right fully bounded ring, $M_{R}$ is finitely generated, $P \in \operatorname{Spec}_{2}(M)$ and $I=\operatorname{ann}_{R}(M / P)$. By [11, Proposition 2.1(ii)], $I$ is a prime ideal of $R$. Thus $R / I$ is a right bounded ring by our assumption. Since now $(M / P)_{(R / I)}$ is finitely generated, $M / P$ is bounded as an $R / I$ module by Proposition 3.1(i), proving that $M_{R}$ is fully bounded. The other direction is clear.

Theorem 3.3. Boundedness is a Morita invariant property.

Proof. Let $R$ and $S$ be Morita equivalent rings with category equivalence $\alpha$ : Mod-R $\rightarrow$ Mod- $S$. We first claim that if $M$ is an $R$-module such that $\operatorname{ann}_{R}(M) \leq_{e} R_{R}$ then $\operatorname{ann}_{S}(\alpha(M)) \leq_{e} S_{S}$. Let $I=\operatorname{ann}_{R}(M)$ and $B=\operatorname{ann}_{S}(\alpha(R / I))$. By [1, Proposition 21.11], $R / I$ is Morita equivalent to $S / B$. Since $M$ is faithful as an $R / I$-module, $B=$ $\operatorname{ann}_{S}(\alpha(M))$ by [1, Proposition 21.6]. Then also $\alpha(R / I)$ is a projective generator in Mod- $S / B$. Thus there exist $L \in \operatorname{Mod}-S / B$ and $n \geq 1$ such that $(S / B) \oplus L=[\alpha(R / I)]^{n}$ in Mod- $S / B$ as well as in Mod- $S$. Now consider the exact sequence $0 \rightarrow I \rightarrow R \rightarrow R / I$. Since Morita equivalences preserve co-kernels and essential monomorphisms, $\alpha(R / I)$ is a co-kernel of an essential monomorphism in Mod- $S$. Hence it is a singular $S$ module. Therefore, $(S / B)_{S}$ is singular and so $B \leq_{e} S_{S}$, as claimed.

Suppose now that $M$ is a bounded $R$-module. It is easy to verify that a module $X_{R}$ is bounded if $\operatorname{ann}_{R}(\operatorname{co-ker} f) \leq_{e} R_{R}$, for every essential monomorphism $f: N_{R} \rightarrow X_{R}$. Thus $(\alpha(M))_{S}$ is bounded by the first part, and the proof is complete. 
COROllary 3.4. Both L-boundedness and right boundedness are Morita invariant properties.

Proof. Note that a module $M_{R}$ is L-bounded if and only if it is bounded as a module over $R / \operatorname{ann}_{R}(M)$. Now let $R$ and $S$ be Morita equivalent rings with category equivalence $\alpha:$ Mod- $R \rightarrow$ Mod- $S$. Suppose that $M_{R}$ is an L-bounded module, $I=\operatorname{ann}_{R}(M)$ and $B=\operatorname{ann}_{S}(\alpha(M))$. As observed in the proof of Theorem 3.3, $(R / I) \stackrel{\alpha}{\approx}(S / B)$. Also $S$ is isomorphic to a direct summand of a finite direct sum of copies $\alpha(R)$ in Mod- $S$. Therefore, the result is obtained by Theorem 3.3 and Proposition 3.1(i).

THEOREM 3.5. The following statements are equivalent for a ring $R$.

(i) $\operatorname{Soc}\left(R_{R}\right) \leq_{e} R_{R}$.

(ii) $R$ is a right bounded ring and the class of bounded $R$-modules is closed under direct sums.

(iii) Every free R-module is bounded.

(iv) Every R-module is bounded.

Proof. (i) $\Rightarrow$ (ii). Let $B=\operatorname{Soc}\left(R_{R}\right)$. Since $B$ lies in all the essential right ideals of $R$, the ring $R$ is right bounded and in $R$, any intersection of essential right ideals is essential. Therefore, as observed in the proof of Proposition 3.1(i), any direct sum of bounded modules is a bounded module.

(ii) $\Rightarrow$ (iii). This is clear.

(iii) $\Rightarrow$ (iv). Apply Proposition 3.1(i) and the fact that any $R$-module is a homomorphic image of a free $R$-module.

(iv) $\Rightarrow$ (i). Let $\left\{I_{\alpha}\right\}_{\alpha \in A}$ be the family of all essential right ideals of $R$. Then we have

$\bigoplus_{\alpha \in A} I_{\alpha} \leq_{e} R^{(A)}$. By our assumption, the $R$-module $R^{(A)}$ is bounded. Thus there exists $I \unlhd_{e} R_{R}$ such that $R^{(A)} I \subseteq \bigoplus_{\alpha \in A} I_{\alpha}$. This shows that $I \subseteq \bigcap_{\alpha \in A} I_{\alpha}=\operatorname{Soc}\left(R_{R}\right)$. Thus (i) holds.

A ring $R$ is said to be right semi-Artinian if for every $I \triangleleft R$, the right socle of the $\operatorname{ring} R / I$ is nonzero (or equivalently, every nonzero $R$-module has a nonzero socle).

Corollary 3.6. A ring $R$ is right semi-Artinian if and only if all $R$-modules are L-bounded.

Proof. $(\Rightarrow)$ Apply Theorem 3.5.

$(\Leftarrow)$. Let $I \unlhd R, M$ be an $R / I$-module and $B=\operatorname{ann}_{R}(M)$. By our assumption, $M_{R}$ is L-bounded and so $M_{R / B}$ is bounded. It follows that $M_{R / I}$ is bounded by Lemma 2.1(vi). Now apply Theorem 3.5 to deduce that the right socle of the ring $R / I$ is an essential right ideal. This shows that $R$ is a right semi-Artinian ring.

It is known that in prime rings the right and left socles coincide. We say that a ring $R$ is pre semi-Artinian if, for every prime ideal $P$ of $R$, the socle of the ring $R / P$ is nonzero (or equivalently, since $R / P$ is a prime ring, an essential (left) right ideal). Note that for every infinite set $\Lambda$ and every field $F$, the von Neumann regular ring $R=F^{\Lambda} / F^{(\Lambda)}$ is pre semi-Artinian but not semi-Artinian because $\operatorname{Soc}\left(R_{R}\right)=0$. 
THeOREM 3.7. All nonzero $R$-modules are fully bounded if and only if $R$ is a pre semiArtinian ring.

Proof. $(\Rightarrow)$ Let $P$ be a prime ideal of $R$ and $T=R / P$. In view of Theorem 3.5, we shall show that every free $T$-module is bounded. Let $F$ be a free $T$-module. By our assumption and Lemma 2.1(vii), $F_{T}$ is fully bounded. On the other hand, $F_{T}$ is $\mathcal{L}_{2}$-prime by Proposition 2.2. Hence $F_{T}$ is bounded, as desired.

$(\Leftarrow)$. This is clear by the definition and Theorem 3.3.

Proposition 3.8. A ring $R$ is right Artinian if and only if $R_{R}$ has Krull dimension, ideals in $R$ are finitely generated as right ideals and all $R$-modules are fully bounded.

Proof. $(\Rightarrow)$ This follows by Theorem 3.5 and the well-known result that a right Artinian ring is right Noetherian with zero Krull dimension.

$(\Leftarrow)$ By [11, Proposition 3.2(ii) and Theorem 3.1] and Theorem 3.5, $R$ is right Artinian.

Remark 3.9. Proposition 3.8 does not hold if we replace 'fully bounded' by 'bounded'. For example, if $R=\left[\begin{array}{ll}\mathbb{Z} & \mathbb{Q} \\ 0 & \mathbb{Q}\end{array}\right]$ then $\left[\begin{array}{ll}0 & \mathbb{Q} \\ 0 & \mathbb{Q}\end{array}\right]=\operatorname{Soc}\left(R_{R}\right) \leq_{e} R_{R}$ and hence all $R$-modules are bounded by Theorem 3.5. But $R$ is not even a right semi-Artinian ring. This example shows also that there exist bounded $R$-modules which are not L-bounded by Corollary 3.6 and Theorem 3.5.

\section{Fully bounded modules with Krull dimension}

Let $R$ be a ring, $M$ an $R$-module. Then according to [9, Corollary 6.2.18], $\mathrm{K} \cdot \operatorname{dim}(M) \leq \mathrm{K} \cdot \operatorname{dim}\left(R_{R}\right)$, provided that both dimensions exist. Also it is known that when $R$ is right Noetherian, Cl.K.dim $(R) \leq \mathrm{K} \cdot \operatorname{dim}\left(R_{R}\right)$, and that the inequality $\mathrm{K} \cdot \operatorname{dim}\left(R_{R}\right) \leq \mathrm{Cl} . \mathrm{K} \cdot \operatorname{dim}(R)$ holds for right FBN rings $R$; see [3, Theorem 15.13]. In this section we prove a theorem which gives classical Krull dimension as an upper bound for the Krull dimensions of certain modules, even if the base ring does not have Krull dimension. In particular, for certain modules $M$ over rings $R$ with Cl.K.dim $(R)<$ $\mathrm{K} \cdot \operatorname{dim}\left(R_{R}\right)$ (for example, if $R=\mathbb{Z} \oplus A$ where $A$ is the $n$th Weyl algebra over $\mathbb{C}$ ), the inequality $\mathrm{K} \cdot \operatorname{dim}(M) \leq \mathrm{K} \cdot \operatorname{dim}\left(R_{R}\right)$ is improved by $\mathrm{K} \cdot \operatorname{dim}(M) \leq \mathrm{Cl} \cdot \mathrm{K} \cdot \operatorname{dim}(R)$. The crucial inequality $\mathrm{K} \cdot \operatorname{dim}\left(R_{R}\right) \leq \mathrm{Cl} \cdot \mathrm{K} \cdot \operatorname{dim}(R)$ for right FBN rings $R$ is also a corollary of our Theorem 4.1.

THEOREM 4.1. Let $R$ be a ring and $M_{R}$ be a nonzero quasi projective fully bounded $\mathcal{L}_{2}$ Noetherian module. Assume that $\mathrm{Cl} . \mathrm{K} \cdot \operatorname{dim}(R)$ and $\mathrm{K} \cdot \operatorname{dim}(M)$ exist. Then $\mathrm{K} \cdot \operatorname{dim}(M) \leq$ Cl.K.dim(R).

Proof. We prove the theorem by induction on Cl.K.dim $(R)$. Since $M_{R}$ is $\mathcal{L}_{2^{-}}$ Noetherian, by [11, Theorem 3.1] there exists an $\mathcal{L}_{2}$-prime submodule $P \unlhd M_{R}$ such that $\mathrm{K} \cdot \operatorname{dim}(M)=\mathrm{K} \cdot \operatorname{dim}\left[(M / P)_{R}\right]$. Let $I=\operatorname{ann}_{R}(M / P)$, which is a prime ideal of $R$. Suppose first that $\mathrm{Cl} \cdot \mathrm{K} \cdot \operatorname{dim}(R)=0$. Hence by hypothesis, $M / P$ is a bounded module 
over the simple ring $R / I$. Thus $M / P$ is a semisimple $R / I$-module with Krull dimension. This shows that $\mathrm{K} \cdot \operatorname{dim}(M)=0$.

Now assume that $\mathrm{Cl} \cdot \mathrm{K} \cdot \operatorname{dim}(R)=\alpha$ and the theorem holds for any ring with classical Krull dimension less than $\alpha$. Let $L=M / P$ and $T=R / I$. We shall show that $\mathrm{K} \cdot \operatorname{dim}\left(L_{T}\right) \leq \alpha$. Notice that $L_{T}$ is quasi projective, $\mathcal{L}_{2}$-Noetherian and fully bounded by Lemma 2.1 and Proposition 3.1(ii). In view of [9, Lemma 6.2.8], it is enough to prove that for all $N \leq_{e} L_{T}, \mathrm{~K} \cdot \operatorname{dim}\left[(L / N)_{T}\right]<\alpha$. Therefore suppose that $N \leq_{e} L_{T}$. Since $M_{R}$ is fully bounded, $L_{T}$ is bounded and so there exists $B \unlhd_{e} T_{T}$ such that $L B \subseteq N$. Again $(L / L B)_{T}$ is $\mathcal{L}_{2}$-Noetherian and so $\mathrm{K} \cdot \operatorname{dim}\left[(L / L B)_{T}\right]=\mathrm{K} \cdot \operatorname{dim}\left[(L / Q)_{T}\right]$ for some $\mathcal{L}_{2}$-prime submodule $Q / L B \unlhd L / L B$. Now $0 \neq B \subseteq \operatorname{ann}_{T}(L / Q):=C$ and so $\mathrm{Cl} . \mathrm{K} \cdot \operatorname{dim}(T / C)<\mathrm{Cl} \cdot \mathrm{K} \cdot \operatorname{dim}(T)$ because $C$ is a nonzero prime ideal in the prime ring $T$. Thus by the induction assumption, $\mathrm{K} \cdot \operatorname{dim}\left[(L / Q)_{T / C}\right] \leq \mathrm{Cl} \cdot \mathrm{K} \cdot \operatorname{dim}(T / C)<\alpha$. This shows that $\mathrm{K} \cdot \operatorname{dim}\left[(L / N)_{T}\right] \leq \mathrm{K} \cdot \operatorname{dim}\left[(L / L B)_{T}\right]<\alpha$, and the proof is complete.

Corollary 4.2. Over a simple ring $R$, every quasi-projective Noetherian selfgenerator fully bounded $R$-module is either Artinian or does not have Krull dimension.

Proof. Apply Theorem 4.1.

Let $A, B$ be rings and $M$ be a left $B$-right $A$-bimodule. It is easy to verify that every prime ideal in the formal triangular matrix $\operatorname{ring} T=\left[\begin{array}{ll}A & 0 \\ M & B\end{array}\right]$ has the form $\left[\begin{array}{ll}P & 0 \\ M & B\end{array}\right]$ or $\left[\begin{array}{ll}A & 0 \\ M & Q\end{array}\right]$ for some prime ideals $P \unlhd A$ and $Q \unlhd B$. It follows that $T$ is a pre semi-Artinian ring if and only if $A$ and $B$ are so. Thus, using formal triangular matrix rings, we are able to construct examples of noncommutative pre semi-Artinian rings $R$ which do not have Krull dimensions; for example, suppose that $R=\left[\begin{array}{cc}F & 0 \\ M\end{array}\right], F$ is a field and $M_{F}$ is nonfinitely generated free. Now by a slight modification of the proof of Theorem 4.1, the following result can be proved which gives an upper bound on the Krull dimension of $\mathcal{L}_{2}$-Noetherian modules over pre semi-Artinian rings (even if the ring may not have Krull dimension).

Theorem 4.3. Let $R$ be a pre semi-Artinian ring and $M_{R}$ be $\mathcal{L}_{2}$-Noetherian. Then $\mathrm{K} \cdot \operatorname{dim}(M) \leq \mathrm{Cl} . \mathrm{K} \cdot \operatorname{dim}(R)$ provided that both dimensions exist.

Corollary 4.4. Let $R$ be a pre semi-Artinian ring with classical Krull dimension $\alpha$. Then $\mathrm{K} \cdot \operatorname{dim}(M) \leq \alpha$ for any Noetherian self-generator module $M_{R}$. In particular, if $\alpha=0$ then every Noetherian self-generator $R$-module is Artinian.

Proof. This follows by Theorem 4.3 and the fact that self-generator Noetherian modules are $\mathcal{L}_{2}$-Noetherian modules with Krull dimensions.

\section{References}

[1] F. W. Anderson and K. R. Fuller, Rings and Categories of Modules, Granduate Texts in Mathematics 13 (Springer, Berlin, 1973).

[2] L. Bican, P. Jambor, T. Kepka and P. Nemec, 'Prime and coprime modules', Fund. Math. 57 (1980), 33-45. 
[3] K. R. Goodearl and R. B. Warfield Jr, An Introduction to Noncommutative Noetherian Rings (Cambridge University Press, New York, 2004).

[4] A. Haghany and M. R. Vedadi, 'Endoprime Modules', Acta Math. Hungar. 106(1-2) (2005), 89-99.

[5] L. Heakyung, 'Strongly right FBN rings', Bull. Aust. Math. Soc. 38(3) (1988), 457-464.

[6] L. Heakyung, 'Right FBN rings and bounded modules', Comm. Algebra 16(5) (1988), 977-987.

[7] L. Heakyung, 'On relatively FBN rings', Comm. Algebra 23(8) (1995), 2991-3001.

[8] T. Kok-Ming, 'Homological properties of fully bounded Noetherian rings', J. Lond. Math. Soc. 2(55)(1) (1997), 37-54.

[9] J. C. McConnell and J. C. Robson, Noncommutative Noetherian Rings (Wiley-Interscience, New York, 1987).

[10] P. F. Smith, 'Modules with many homomorphisms', J. Pure Appl. Algebra 197(1-3) (2005), 305-321.

[11] M. R. Vedadi, ' $\mathcal{L}_{2}$-prime and dimensional modules', Int. Electron. J. Algebra 7 (2010), 47-58.

[12] I. E. Wijayanti and R. Wisbauer, 'On coprime modules and comodules', Comm. Algebra 37(4) (2009), 1308-1333.

\section{A. HAGHANY, Department of Mathematical Sciences, Isfahan University of Technology, Isfahan 84156-83111, Iran e-mail: aghagh@cc.iut.ac.ir}

\section{MAZROOEI, Department of Mathematical Sciences,} Isfahan University of Technology, Isfahan 84156-83111, Iran e-mail: m.mazrooei@math.iut.ac.ir

M. R. VEDADI, Department of Mathematical Sciences, Isfahan University of Technology, Isfahan 84156-83111, Iran e-mail: mrvedadi@cc.iut.ac.ir 